\title{
TESTS EN HUMANIDADES: UNA EVALUACION FUZZY
}

\author{
Pablo C. Rovarini Díaz \\ Universidad Tecnológica Nacional, FRT Departamento de Sistemas \\ Tucumán, Argentina, 4000 \\ provarini@gmail.com \\ and \\ María de las Mercedes Cerviño \\ Universidad Tecnológica Nacional, FRT Departamento de Ciencias Básicas \\ Tucumán, Argentina, 4000 \\ mercervino@gmail.com
}

Proyecto de Investigación UTILIZACION DE REDES SOCIOECONÓMICAS EN TOMA DE DECISIONES

\begin{abstract}
In this paper we follow a research path based on the incorporation of concepts from Fuzzy Logic trying to find a fair and balanced qualification in student's evaluation. Our approach tries to solve this problem, particularly for those students which focus its interest in humanistic area, but not being restrictive to them in any sense. Our proposal is developed and supported on a questionnaire with closed answers. We have adopted this form because we center our interest in groups of large size.
\end{abstract}

Keywords: Fair evaluation, Fuzzy Logic, evaluator profile

\section{Resumen}

En este trabajo seguimos una línea de investigación que incorpora conceptos de Lógica Fuzzy a la tarea de encontrar una calificación justa y equilibrada para la evaluación de estudiantes. Nuestro análisis trata el problema particularmente sobre los que desarrollan sus actividades en disciplinas humanísticas, no siendo de ninguna manera restrictivo a ellas. Nuestra propuesta de solución está desarrollada sobre la base de un cuestionario con respuestas cerradas, forma que hemos adoptado debido a que centramos nuestro interés en grupos de gran tamaño.

Palabras claves: Evaluación justa, Lógica Fuzzy, perfil de evaluador 


\section{1 - Introducción}

La evaluación de estudiantes es una de las facetas más importantes en sistemas educacionales. En los últimos años encontramos una buena cantidad de trabajos [7, 2, 1entre muchos otros] sobre cómo evaluar test de estudiantes para determinar su grado de aprovechamiento sobre temas desarrollados en un curso determinado, intentando medir los conocimientos adquiridos y asignando un grado como resultado. Esta evaluación, en general, resulta compleja. Definimos un test de evaluación como un conjunto de preguntas que se presentan al estudiante para que las responda dentro de un límite de tiempo previamente determinado. Para lograr una discusión beneficiosa a nuestros fines, es adecuado dividir las características de la prueba en dos tipos básicos:

- Conjunto de preguntas con respuestas abiertas
- Conjunto de preguntas con respuestas cerradas

En este trabajo presentamos un nuevo método aplicable al segundo caso, para lo que vamos a necesitar la definición de algunos términos. Un test de evaluación $\mathrm{V}$ está compuesto por p preguntas. Cada una de ellas admite $\mathrm{n}$ respuestas (la generalización a un número diferente de respuestas a cada pregunta es trivial), usando la siguiente notación:

$$
\begin{array}{lll}
\mathrm{V}=\left\{\mathrm{v}_{1}, \mathrm{v}_{2}, \ldots, \mathrm{v}_{\mathrm{p}}\right\} & ; & \mathrm{v}_{\mathrm{i}}: \mathrm{i} \text {-esima pregunta } \\
\mathrm{vi}=\left\{\mathrm{a}_{\mathrm{i} 1}, \mathrm{a}_{\mathrm{i} 2}, \ldots, \mathrm{a}_{\mathrm{in}}\right\} & ; & \mathrm{a}_{\mathrm{ij}} \text { : respuesta } \mathrm{j} \text {-esima a la pregunta i-esima }
\end{array}
$$

Como diseñadores del mismo, nuestro primer paso al construir un test es escoger $\mathrm{p}$ preguntas, proveyendo a cada una de ellas con un conjunto de $\mathrm{n}$ respuestas. En este momento aparece un problema directamente asociado al tipo de respuestas cerradas. La teoría de conjuntos tradicional solo nos ofrece la magra posibilidad de considerar a cada respuesta dentro de un conjunto sumamente restringido de valores: [0,1]. En otras palabras, el encargado de tomar la decisión si la respuesta es correcta o no, solo podrá establecer dos valores, CIERTO o FALSO, lo que en una buena cantidad de casos resulta totalmente inexacta, ineficiente e insuficiente. El caso general nos lleva a considerar posibilidades reales un tanto más complicadas que ofrecerle al decisor respuestas falsas y una y solo una totalmente cierta. En el ámbito de las ciencias exactas, una situación de este último tipo es relativamente frecuente. Pero una situación totalmente distinta ocurre en el contexto de las ciencias humanísticas (fillosofía, historia, psicología, etc.). Debemos entender que el grado de verdad de una respuesta en este ámbito es dependiente de factores humanos, como por ejemplo ocurre cuando la pregunta es:

\section{¿Por qué el General X hizo tal cosa?}

Posiblemente la respuesta sea diferente para dos historiadores (uno de ellos decidido partidario de la conducta política del General X y el otro un opositor recalcitrante). Como nota acertadamente Radisnky [7], la naturaleza dialéctica de los problemas y sus características en humanidades no permite, en general, respuestas totalmente ciertas o totalmente falsas (excepto para preguntas primitivas, como por ejemplo: ¿Qué día se declaró la independencia argentina?). Esta falta de precisión provocada por diferentes enfoques, sugiere que el diseñador del test solicite opiniones a expertos en el tema intentando llegar a una opinión colectiva suficientemente consensuada. La intención, como debe entenderse de lo anteriormente expuesto, es lograr como objetivo coherente un resultado (respuestas) que reflejen diferentes puntos de vista y no castiguen a un estudiante por no compartir las creencias de su eventual evaluador. Queda claro entonces que precisamos de un sustento matemático más fuerte.

Recordemos que Zadeh [8] indicó que la Teoría de Conjuntos Fuzzy tiene como finalidad la representación de cómo la mente humana percibe y manipula la información:

"Indeed the pervasiveness of fuzziness in human thought suggests that much of the logic behind reasoning is not the traditional logic, but a logic with fuzzy truths, fuzzy connections, fuzzy rules of inference"

Es interesante tomar como premisa que los seres humanos utilizan frecuentemente conceptos fuzzy cuando perciben el entorno que los rodea y cuando piensan en procura de soluciones a sus problemas, apareciendo variables lingüísticas y modificadores como bueno, alto, similar, muy, bastante, cercano, etc. Entonces aceptemos que una de las características más representativas del pensamiento humano es la de lograr una síntesis de la información en niveles de conjuntos fuzzy para describir lingüísticamente situaciones particulares. Las ideas que impregnan a los conjuntos fuzzy y sus valores de pertenencia proveen de un excelente modelo para conceptos complejos y muy difíciles de cubrir con otras representaciones, siendo totalmente eficientes en representar el conocimiento necesario para lograr decisiones efectivas y acertadas. Los procesos dinámicos de nuestro cerebro son de una gran complejidad, lo que hace que los procesos de toma de decisiones exactas, a partir de una mezcla heterogénea de modificadores y su transformación a valores numéricos, resulte epistemológicamente imposible a menos que la 
tratemos mediante herramientas adecuadas, como la lógica fuzzy. Siguiendo a Zadeh, sugerimos que encontrar cada vez mejores métodos de evaluación no es nada más que procurar aproximaciones fuzzy.

En la sección 2 de este trabajo damos nuestra visión de cómo componer opiniones contradictorias mediante lógica fuzzy siguiendo ideas de Cox [3], dejando para la sección 3 una variante del método de Biswas [1] sobre cómo hacer para obtener una graduación razonable para un test.

\section{2 - Composición de Opiniones y Creación de Perfiles}

Partiendo del problema planteado a un diseñador de evaluaciones al enfrentar opiniones disímiles sobre un mismo tema, no cuesta demasiado asimilarlo al correspondiente a integrar opiniones contradictorias en el ámbito de la economía [5]. Para esto asumimos que vamos a utilizar reglas condicionales del tipo IF-THEN, con una estructura:

$$
<\text { antecedente }>\rightarrow<\text { consecuente }>
$$

Con $\operatorname{var}_{i}, \mathrm{i}=1, \ldots, \mathrm{k}$, nombramos las variables de entrada involucradas, con val $_{\mathrm{i}}$ al valor que dichas variable toman sobre sus respectivos dominios de definición, y con $\mathrm{z}$ a una variable de salida asociada a la regla:

$$
\text { IF }<\operatorname{var}_{1}>\text { IS }<\operatorname{val}_{1}>\text { AND } \ldots \text { AND }<\operatorname{var}_{\mathbf{k}}>\text { IS }<\text { val }_{\mathbf{k}}>\text { THEN z IS }<\text { val }_{\mathrm{z}}>
$$

para expresar las diferentes opiniones, permitiendo que tales reglas eventualmente carezcan del condicionante reglas sin antecedente - transformándose en lo que denominamos una regla incondicional o hecho. Podemos verlas como sentencias de relaciones fuzzy que utilizan proposiciones incondicionales. Puesto que una regla incondicional no posee antecedente, se toma como totalmente cierta, con una credibilidad 1.0. El modelo solicita que las reglas incondicionales se apliquen secuencialmente en el proceso de agregación mediante un operador lógico AND (en este trabajo usaremos la definición de Zadeh, asociándolo con el operador MIN) entre la región de salida fuzzy en el momento que se dispara la regla y el conjunto fuzzy asociado con la regla incondicional, lo que se realiza tomando el mínimo de los valores de pertenencia para cada punto del dominio (a menos que la región de salida sea vacía, caso en que simplemente tomamos el conjunto fuzzy asociado al consecuente como nueva región de salida). Cuando utilizamos reglas incondicionales, la Base de Conocimiento fuzzy no representa más a un conjunto de reglas declarativas, lo que significa que el orden de ejecución de reglas es importante, y no está determinado por el motor inferencial. Para las reglas condicionales, usaremos un operador OR (también de acuerdo con Zadeh, asociándolo con el operador MAX). Además, todas las reglas incondicionales deben ejecutarse antes de cualquier regla condicional, ya que estas reglas incondicionales proveen un conjunto soporte por defecto para el modelo si no se dispara ninguna regla condicional.

Vamos a seguir pautas semejantes, con los cambios adecuados, al tratamiento de reglas incondicionales señaladas en trabajos anteriores para realizar el diseño del modelo para el caso bajo tratamiento, condensados en un trabajo de uno de los autores [6]. Para esto, comenzamos con las reglas incondicionales, en un orden un tanto arbitrario, ya que estamos considerando que todas las opiniones revisten la misma importancia (mas adelante consideraremos una variante producida por la incorporación de la importancia relativa de cada regla). Para mostrar la metodología, hemos desarrollado un algoritmo en pseudocódigo, mostrado en Figura 1.

En este algoritmo, agreginc y agregcon representan dos funciones que nos permite realizar un proceso de agregación entre dos conjuntos fuzzy. (el operador MIN y el operador MAX, respectivamente).

Nuestro foco de interés reside en la tarea de evaluación, y recordemos que un test compuesto por un conjunto de preguntas con un cierto grado de subjetividad al estar formuladas en un lenguaje inherentemente ambiguo (como cualquier lenguaje coloquial), causa buena parte de los problemas que tratamos de resolver. La variable que representa resultados en el algoritmo de Figura 1, toma como dominio una escala de evaluación para cada respuesta compuesta por cinco grados, propuesta por Biswas $[1,2]$, pero que puede cambiarse a cualquier otra sin mayores problemas:

$$
\left.\left\{\begin{array}{c}
\text { A excelente } \\
\text { B muy buena } \\
\text { C buena } \\
\text { D Satisfactoria } \\
\text { E Insatisfactoria }
\end{array}\right\} \text { Valores cualitativos } \quad \begin{array}{l}
A=5 \\
B=4 \\
C=3 \\
D=2 \\
E=1
\end{array}\right\} \text { grado }
$$

Tomaremos como representativa de cada una de estas alternativas a funciones de pertenencia mostradas en Figura 2, y descriptas por este autor mediante los siguientes vectores:

Excelente $[E X]=\{0 / 0,0 / 20,0.5 / 40,0.8 / 60,1 / 80,1 / 100\}=\{0,0,0.5,0.8,1,1\}$ 
Muy Bueno $[\mathrm{MB}]=\{0,0,0.6,0.9,0.9,0.6\}$

Bueno $[\mathrm{BU}]=\{0.0 .2,0.8,0.9,0.6 .0 .2\}$

Satisfactorio $[\mathrm{SA}]=\{0.4,0.5,0.9,0.5,0.2,0\}$

Insatisfactorio $[\mathrm{IN}]=\{1,1,0.4,0.2,0,0\}$

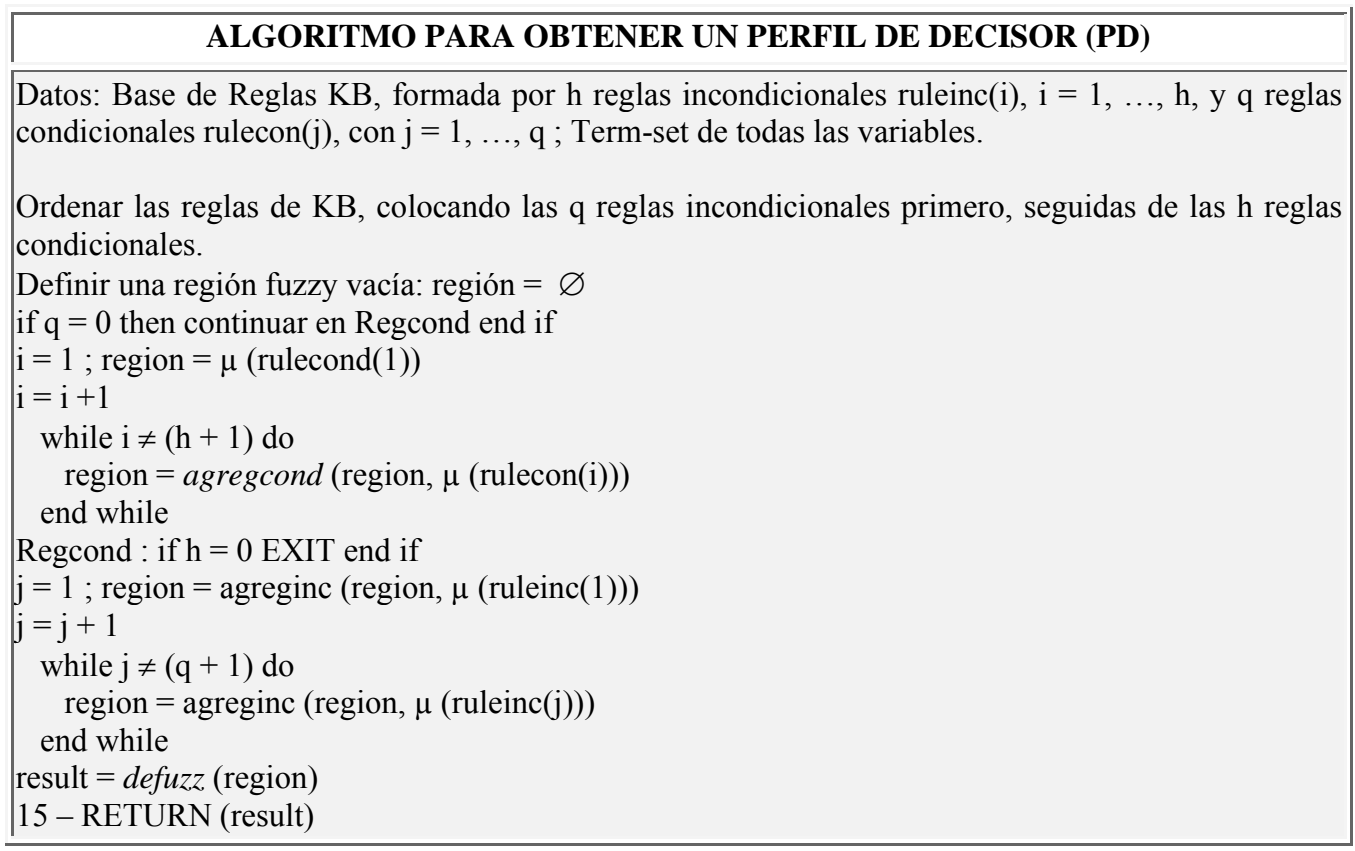

Fig, 1 - Algoritmo de agregación

Supongamos tener $\mathrm{D}$ decisores, representativos de los expertos consultados. Cada pregunta $\mathrm{r}, \mathrm{r}=1, \ldots, \mathrm{p}$, genera en cada decisor $\mathrm{n}$ respuestas en la escala anterior $(\mathrm{n}=5$ siguiendo a Biswas) y mediante agregación obtenemos un patrón o perfil de respuestas para la pregunta en cuestión según el experto considerado. Este proceso de agregación se formaliza en el algoritmo PD mostrado en la Figura 1.

Resumiendo, se presenta a cada decisor una pregunta $\mathrm{r}$ con $\mathrm{n}$ respuestas. Es tarea del decisor emitir su opinión sobre las respuestas propuestas para ella, dentro de la escala ya descripta. Componemos estas respuestas según el Algoritmo PD y obtenemos un perfil para la pregunta $\mathrm{r}$ según el experto i-esimo. Como trabajamos con D expertos y p preguntas, creamos así una base de información de perfiles con $(D * p)$ entradas. En el caso que queramos obtener una apreciación colectiva o grado de la pregunta $\mathrm{p}$ sobre los $\mathrm{D}$ evaluadores simultáneamente, combinamos estos perfiles (correspondientes a región en el Algoritmo PD). Este trabajo se logra mediante una defuzzificación previa, con lo que obtenemos el valor nítido result para cada decisor, afectado por la importancia relativa de cada uno de ellos mediante el parámetro $\eta(0 \leq \eta \leq 10)$, y la ayuda de la función:

$$
\operatorname{grado}(i)=\operatorname{COG}\left[\eta_{1} \text { result }(1) \ldots \eta_{D} \text { result }(D)\right] ; \mathrm{i}=1 . . \mathrm{p}
$$

Donde el cálculo del centro de gravedad COG puede reemplazarse por cualquier estrategia para encontrar un único valor (nítido) para la pregunta $\mathrm{p}$, sus $\mathrm{n}$ respuesta y la opinión de los decisores consultados. Un ejemplo se muestra en la Figura 3, donde hemos asumido que el evaluador 2 respondió a la calificación de las $\mathrm{n}$ respuestas de la manera siguiente:

$$
1 \rightarrow \mathrm{BU} ; 2 \rightarrow \mathrm{EX} ; 3 \rightarrow \mathrm{BU} ; 4 \rightarrow \mathrm{IN} ; 5 \rightarrow \mathrm{MB} \text {, con } \eta=2.0
$$

con lo que encontramos un valor de 45 para la valoración de la pregunta en su totalidad, lo que muestra que el Decisor 2 considera personalmente que esta pregunta es BUENA. Notemos que una heurística utilizada en la evaluación consiste en permitir una tasa de repetición igual a dos, tanto para expertos como para estudiantes (en otras palabras, se puede repetir un valor solo dos veces). Después de la aplicación del algoritmo, el resultado colectivo para los expertos consultados (en el ejemplo seis de ellos) es de 62 para la pregunta r, lo que indica que el 
conjunto de todos los evaluadores consultados deciden que se trata de una MUY BUENA pregunta (también mostrado en la Figura 3).

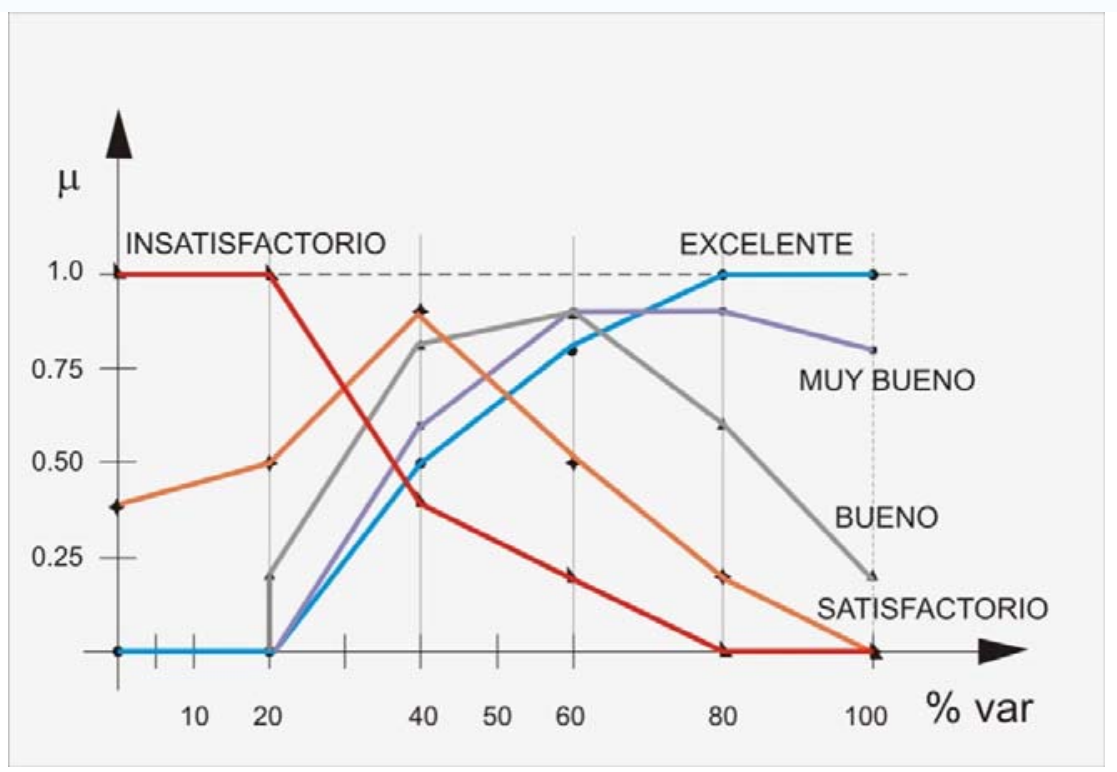

Fig. 2 - Funciones de pertenencia consideradas

\section{3 - Determinación del grado de un test}

Partimos ahora del test ya confeccionado, evaluado por expertos y con perfiles de sus preguntas determinados adecuadamente. El siguiente paso es entregar el test a un estudiante y solicitarle una acción en un todo igual a cuando se lo entregamos a un experto. Como resultado de esta acción, el estudiante responde a un determinado número de preguntas (no necesariamente todas), esperando su evaluación y el grado que le corresponde. El encargado final de la evaluación de este test debe realizar diferentes acciones:

1 - Evaluar el test resolviendo si el mismo es suficiente para aprobar la asignatura que lo produjo.

2 - Asignar un grado al mismo.

3 - Justificar tal grado, y simultáneamente utilizar los perfiles de los expertos con la finalidad de aconsejar al estudiante para que se ponga en contacto con el o los expertos que sostienen posturas disímiles y obtener así una mayor comprensión del tema, profundizando aspectos que de otra manera no se detectarían de modo simple.

Estamos absolutamente seguros que el punto 3 anterior reviste una importancia fundamentalmente en la formación de un estudiante, ya que le permite conocer otros puntos de vista, teorías que quizás le resulten nuevas y sobre todo, una mayor cobertura de los temas que trata el test con la finalidad de lograr una meta importante, cual es la de entregar una visión lo más completa posible de un área dentro de la asignatura evaluada.

¿Cómo procede entonces el evaluador del test? Para comenzar, necesitamos una métrica que le permita satisfacer el primer punto. Para esto, resulta adecuado definir lo que se conoce como similitud entre conjuntos fuzzy. Comencemos viendo algunos conceptos matemáticos sencillos involucrados.

El producto escalar o producto interno de dos vectores estándar del espacio euclídeo ortonormal parte presuponiendo una dimensión común a ambos $A=\left[a_{1}, a_{2}\right.$, $\left.\ldots, a_{n}\right]$ y $B=\left[b_{1}, b_{2}, \ldots, b_{n}\right]$. Lo definimos como sigue:

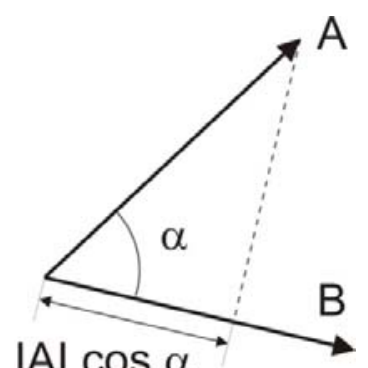

$$
A \cdot B=\sum_{i=1}^{n} a_{i} b_{i}=a_{1} b_{1}+a_{2} b_{2}+\ldots+a_{n} b_{n}
$$

con un interpretación geométrica simple: $\mathrm{A} \cdot \mathrm{B}=|\mathrm{A}||\mathrm{B}| \cos (\alpha)$ 


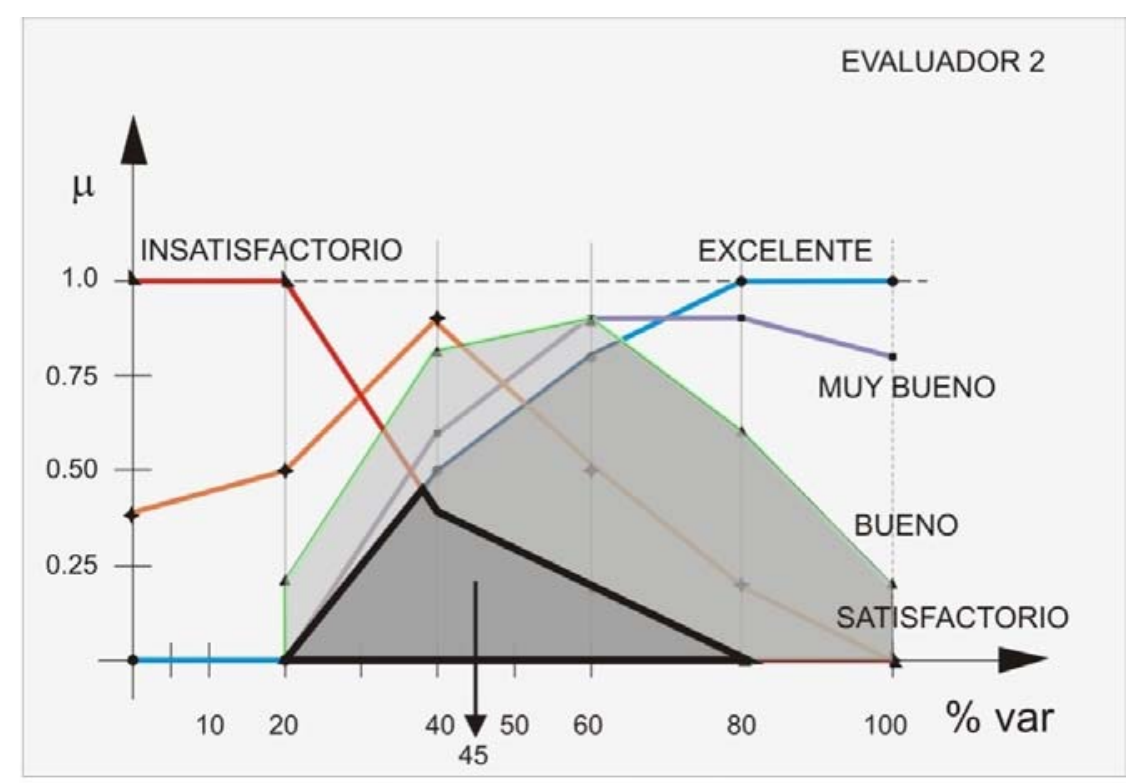

PERFIL DEL EVALUADOR 2

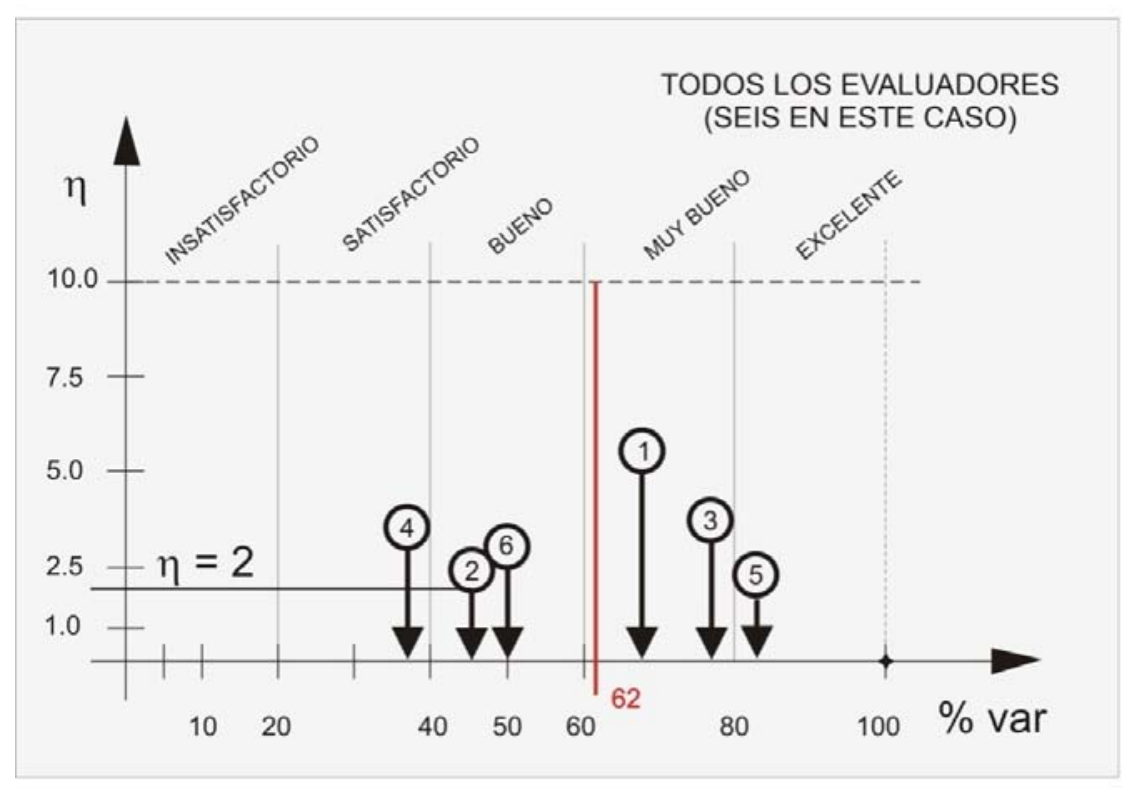

\section{RESULTADO COLECTIVO (pregunta v)}

Fig. 3 - Creación de Perfiles y evaluación colectiva de una pregunta

Siendo $|\mathrm{A}| \cos (\alpha)$ la proyección escalar de A sobre B y $\alpha$ el ángulo entre ellos. La definición formal de un conjunto fuzzy A toma como base un conjunto nítido X asignando un grado de pertenencia a cada elemento de $\mathrm{X}$ como sigue:

$$
\left.A=\left\{\mu_{A}(x), x\right): x \in X\right\}
$$

Donde $\mu_{A}(x) \rightarrow[0,1]$ es un transformación denominado función de pertenencia del conjunto A, siendo $\mu_{A}(x)$ el grado de pertenencia de $\mathrm{x} \in \mathrm{X}$ en A. Es costumbre escribir la ecuación anterior como:

$$
\left.A=\left\{\mu_{A}(x) / x\right): x \in X\right\}
$$


Y simplificando la notación $\mathrm{A}=\mu_{\mathrm{x}}, \mathrm{x} \in \mathrm{X}$. El grado de similitud entre dos conjuntos fuzzy A y B se nota con simil $(\mathrm{A}, \mathrm{B})$ y se define como:

$$
\operatorname{simil}(A, B)=\frac{\boldsymbol{A} \cdot \boldsymbol{B}}{\max (\boldsymbol{A} \cdot \boldsymbol{A}, \boldsymbol{B} \cdot \boldsymbol{B})}
$$

Donde tanto a $\mathbf{A}$ como $\mathbf{B}$ representan vectores con un número de componentes dados por la dimensión del espacio considerado. Si A y $\mathbf{B}$ no son iguales, simil $(\mathbf{A}, \mathbf{B}) \neq 1$, y cuando mayor sea la diferencia, decimos que existe mayor disimilitud ente A y B. Esta medida de la similitud entre dos conjuntos fuzzy utilizando el producto escalar es una buena alternativa a nuestros fines.

Partiendo del test $T$ resuelto por un estudiante y el conjunto de perfiles de los expertos, notados $P_{j}, j=1, \ldots, D$, encontramos la similitud entre los pares $\left(\mathrm{T}, \mathrm{P}_{\mathrm{j}}\right)$ con la intención de realizar una evaluación de $\mathrm{T}$ que, en primera instancia, le asegure al estudiante que será evaluado por un experto con pautas lo más próximas a las suyas.

Para mostrar un ejemplo, tomemos los resultados de un evaluador y un estudiante sobre una pregunta $\mathrm{v}$, tal como se muestra en las Figuras 4 y 5 . El estudiante responde:

$$
1 \rightarrow \mathrm{BU} ; 2 \rightarrow \mathrm{MB} ; 3 \rightarrow \mathrm{SA} ; 4 \rightarrow \mathrm{IN} ; 5 \rightarrow \mathrm{MB}
$$

Y supongamos que el evaluador coincide con el experto 2 anterior. Vemos que hay una gran similitud entre los resultados (valores 45 y 42). Por razones de espacio, solo vamos a calcular el grado de similitud entre lo que sostiene el experto $\mathrm{k}$ y el estudiante con su test $\mathrm{T}$, partiendo de los vectores de cada uno de ellos, coincidentes con las Figuras 4 y 5.

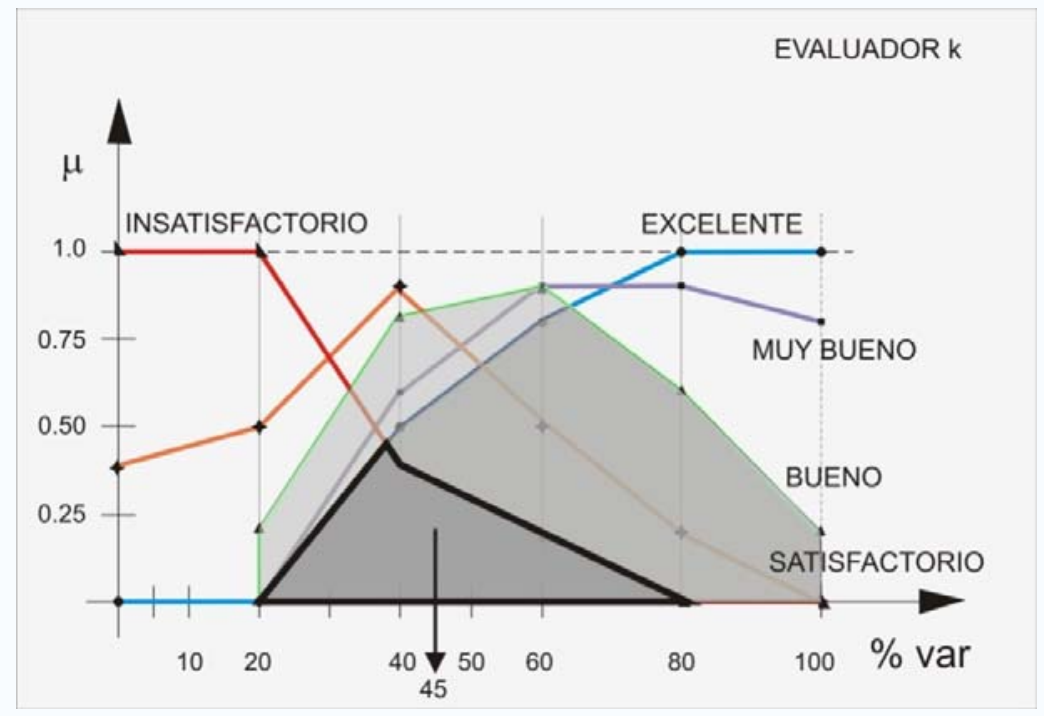

Figura 4 - Importancia de la pregunta v según el Evaluador k

$$
\begin{array}{lll}
(0,0,0.4,0.2,0,0) & \rightarrow & \text { Evaluador k } \\
(0,0,0.4,0.2,0,0) & \rightarrow & \text { Estudiante con T }
\end{array}
$$

En este caso el grado de similitud es muy simple, ya que los dos vectores coinciden por lo que:

$$
\operatorname{simil}(k, T)=1
$$

Lo que nos indica que, en principio, esta pregunta $\mathrm{v}$ incide sobre el grado final en un factor sencillo: directamente el valor asignado a la importancia de la pregunta por el evaluador $\mathrm{k}$. En general, lo que hacemos es afectar al valor de la pregunta (en este caso 45) según el experto con mayor similitud a T (en este caso 42) mediante una ecuación:

$$
\text { Grado Ponderado }(k)=\sigma *(\text { valor de la pregunta })_{k}
$$

Donde $0 \leq \sigma \leq 1$ es una constante que pondera de manera más fina la disimilitud entre el experto $\mathrm{k}$ y el estudiante (en el caso del ejemplo tal disimilitud es muy pequeña, de manera que podemos tomar $\sigma=1$ sin un error apreciable, 
con lo que el grado para el estudiante autor del test $\mathrm{T}$ es de 45). Este proceso debe realizarse sobre todas las preguntas, con lo que obtendremos un grado total, encontrado sencillamente mediante la suma de los grados parciales:

$$
\text { Grado Total }=\sum_{i=1}^{p} \text { Grado Ponderado }(i)
$$

Como esta sumatoria no tiene por qué sumar 100, normalizamos el resultado de la siguiente manera:

$$
\text { Grado Total Normalizado }=\text { Grado Total } / \sum_{i=1}^{p} \text { valor de la pregunta }(i)
$$

Con un resultado en una escala de 1 a 100 con un óptimo igual a 100 (máximo de la escala).

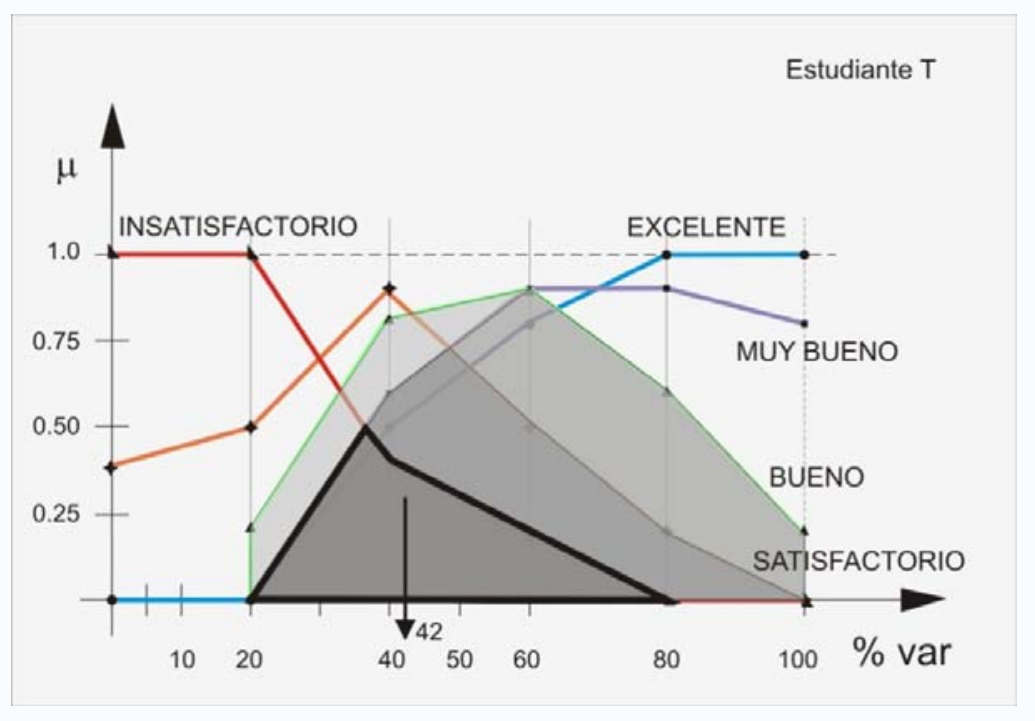

Figura 5 - Resultado del Test para la pregunta $\mathrm{v}$

Una alternativa válida para el cálculo del Grado Total la encontramos si en lugar de trabajar con el valor producido por el experto con mayor similitud con el estudiante, utilizamos el valor obtenido para cada pregunta mediante el método de composición entre los $\mathrm{D}$ expertos mostrado en la sección 2. Una manera muy rápida de encontrar un resultado se logra introduciendo un parámetro $\delta$ que tome en cuenta el error entre la respuesta de un estudiante y el valor de la pregunta consensuado. En las figuras 3 y 5 encontramos valor $(\mathrm{r})=42$ para la evaluación agregada de las respuestas correspondientes a la pregunta $\mathrm{r}$ efectuada por el estudiante durante el test $\mathrm{T}$, y el valor $_{\text {COLECTIVO }}(\mathrm{r})=$ 62, como evaluación colectiva de la bondad de la misma pregunta $r$. Trabajaremos con la distancia entre valor $\mathrm{r}_{\mathrm{T}}(\mathrm{r}) \mathrm{y}$ valor $_{\text {COLECTIvo }}(\mathrm{r})$ llamándola $\delta(\mathrm{r})$ con lo que:

$$
\delta(r)=\mid \text { valor }_{\text {COLECTIVO }}(r)-\operatorname{valor}_{T}(r) \mid
$$

De modo que $\delta(\mathrm{r})$ representa el error entre el valor de la respuesta consensuada por los D expertos y el resultado de las respuestas del estudiante en T, y para este ejemplo resulta $\delta(r)=62-42=20$. Para calcular el grado total del estudiante según su test $\mathrm{T}$ en una escala de 1 a 100, normalizamos la suma de los valores absolutos sobre todas las preguntas:

$$
\operatorname{GTNC}=\left[\sum_{i=1}^{p}\left(\text { valor colectivo }(i)-\sum_{i=1}^{p} \delta(i) / \sum_{i=1}^{p} \text { valor colectivo }(i)\right] * 100\right.
$$

Con la obtención del Grado Total Normalizado Colectivo (GTNC) completamos nuestra evaluación del test T. 


\section{4 - Conclusiones}

En este trabajo hemos presentado una metodología nueva y de modo alguno convencional para evaluar pruebas en humanidades, de acuerdo a dos criterios:

* Similitud entre respuesta de un estudiante-opinión de expertos

* Relación entre los valores consensuados para cada pregunta con el resultado del estudiante

Ambos fueron probados en pequeña escala obteniéndose excelentes resultados, pero sujetos a comprobaciones masivas para validar sus resultados. Hemos desarrollado también un sistema software como soporte de esta metodología, basando nuestro diseño del sistema en el lenguaje formal OZS [4] e implementado en JAVA, particularmente útil cuando el número de estudiantes es elevado (rogamos a los interesados ponerse en contacto con los autores).

Resulta sumamente interesante comparar el Grado Total Normalizado con el Grado Total Normalizado Colectivo, intentando encontrar respuestas a preguntas fundamentales, como cuestionar la agregación o la ponderación de los resultados de los expertos. En este sentido estamos aun en un período de investigación temprana, y esperamos mostrar nuestros resultados en un próximo trabajo.

\section{Bibliografía}

[1] Biswas, Ranjit. An Application of Fuzzy Sets in Student Evaluation. Fuzzy Sets and Systems, Elsevier (Holland), Vol 74, pp. 187-194, 1995.

[2] Chen, Shyi-Ming and Lee, Chia-Hoang. New Method for Student Evaluation Using Fuzzy Sets. Fuzzy Sets and Systems, Elsevier (Holland), Vol 1004, pp 209-218, 1999.

[3] Cox, Earl. Fuzzy Modeling and Genetic Algorithms for Data Mining and Exploration. Morgan Kaufmann Publisher, Elsevier Inc., 2005.

[4] Gruer, Juan Pablo, Rovarini Pablo Cesar et al. Heterogeneous Formal Specification Based on OBJECT-Z and STECHARTS: Semantics and Verification. The Journal of Systems and Software, Helsevier (Holland), January 13, 2004.

[5] Rovarini, Pablo Cesar, Fanjul, Roberto and Figueroa Mario. Determinación del Precio de Venta de un Producto Mediante Toma de Decisiones Fuzzy. Revista Ciencia Tecnología y Medio Ambiente, Año VII, Número 7- ISSN 1667 - 457X, FRT, UTN, pp. 9 a 16, 2008.

[6] Rovarini, Pablo Cesar, Fanjul, Roberto and Juarez, Gustavo. The Fuzzy Control Algorithm. Laboratory Report 1-06, Laboratorio de Inteligencia Artificial UNT, Fall 2006.

[7] Rudenskiy, Igor Davidovich. Fuzzy Knowledge Evaluation Model as a Methodological Basis for Automation of Pedagogical Testing. IEEE Transactions on Education, Vol 50, Nro. 1, pp. 68-73, 2007.

[8] Zadeh, Lotfi. Fuzzy Sets. Information and Control, Vol 8, pp. 338-353, 1965. 